\title{
A Rare Case of Urachal Adenocarcinoma: Long Term Follow Up
}

\author{
Mohammad Reza Darabi ${ }^{1}$, Ali Reza Khoei ${ }^{2}$, Atena Aghaee ${ }^{3}$ and Salman Soltani ${ }^{4,}{ }^{*}$ \\ ${ }^{1}$ Medical Sciences Faculty, Mashhad University of Medical Sciences, Mashhad, Iran \\ ${ }^{2}$ Department of Pathology, Faculty of Medicine, Mashhad University of Medical Sciences, Mashhad, Iran \\ ${ }^{3}$ Nuclear Medicine Research Center, Mashhad University of Medical Sciences, Mashhad, Iran \\ ${ }^{4}$ Department of Urology, Kidney Transplantation Complications Research Center, Mashhad University of Medical Sciences, Mashhad, Iran \\ "Corresponding author: Kidney Transplantation Complications Research Center, Mashhad University of Medical Sciences, Mashhad, Iran. Tel: +98-9153222804, Email: \\ soltanis@mums.ac.ir
}

Received 2018 June 29; Revised 2018 August 11; Accepted 2018 August 21.

\begin{abstract}
Introduction: Urachal adenocarcinoma is an invasive and rare bladder carcinoma that usually presents at advances stages with poor prognosis. The symptoms often include abdominal pain and hematuria. The standard treatment method is surgical excision of the tumor and sometimes, in advanced cases, chemotherapy is needed, too.

Case Presentation: We present a 35-year-old male patient, who referred with acute onset of painless macroscopic hematuria. Pelvic CT scan showed a large mass on the bladder dome, extending to the umblicus. Biopsy of the mass confirmed that the pathologic diagnosis was urachal adenocarcinoma. The patient was then treated surgically with partial cystectomy approach.

Conclusions: Despite microscopic involvement of pelvic lymph nodes, adjuvant therapy was not intended. There were no local recurrence or distant metastasis in long term close follow up.
\end{abstract}

Keywords: Urachal Carcinoma, Urinary Bladder Neoplasm, Signet Ring Cell Carcinoma, Bladder

\section{Introduction}

The urachus is the remnant of urogenital sinus. It often closes spontaneously by the second trimester, leaving the median umbilical ligament as its remnant (1). However, its relation with the urinary bladder may persist in some adult patient. Urachal cancers are rare malignancies of the bladder, which consist of less than $1 \%$ of all bladder cancers, and about 30\% of primary adenocarcinomas (2). The most common type of urachal carcinoma is adenocarcinoma (89\%), followed by sarcoma, and transitional cell carcinoma (4\%). The common symptoms are hematuria and lower abdominal pain (3). Computed tomographic (CT) scan usually shows a midline mass between umbilicus and bladder, which may contain peripheral calcifications in $50 \%-70 \%$ of cases. This pattern is considered as pathognomonic for urachal adenocarcinoma (4). We report a 35-year-old man with stage pT4a urachal adenocarcinoma (5) and microscopic involvement of the pelvic lymph nodes. He was treated with surgical excision, in a partial cystectomy approach, without adjuvant therapies. In the period of 2 years after surgery, no local recurrence or distant metastasis was noticed.

\section{Case Presentation}

A 35-year-old male patient, with no remarkable past medical and family history was presented to our urology clinic with painless macroscopic hematuria for the last six months. In addition, he also had a history of urinary urgency and frequency. His physical examination revealed mass sensation at the suprapubic region. His serum hemoglobin was $14.3 \mathrm{~g} / \mathrm{dL}$, renal function test was normal (his serum creatinine level was 1.1), and the urine was sterile.

Ultrasonography (US) revealed heterogeneous, an irregular mass on the bladder roof. A CT scan showed an irregular mass extending from the superior wall of the bladder, which enhanced after injection of contrast agent (Figure 1).

Subsequently, cystoscopy was performed and showed a solid tumor, which measured about 3 centimeters in the bladder dome. Biopsy of the bladder mass revealed adenocarcinoma and partial cystectomy was planned for treatment. The CT of chest, abdomen, and pelvis did not show any regional or distant metastasis. Open partial cystectomy, with total urachectomy up to the umbilicus and bilateral pelvic node dissection, was done through a midline 

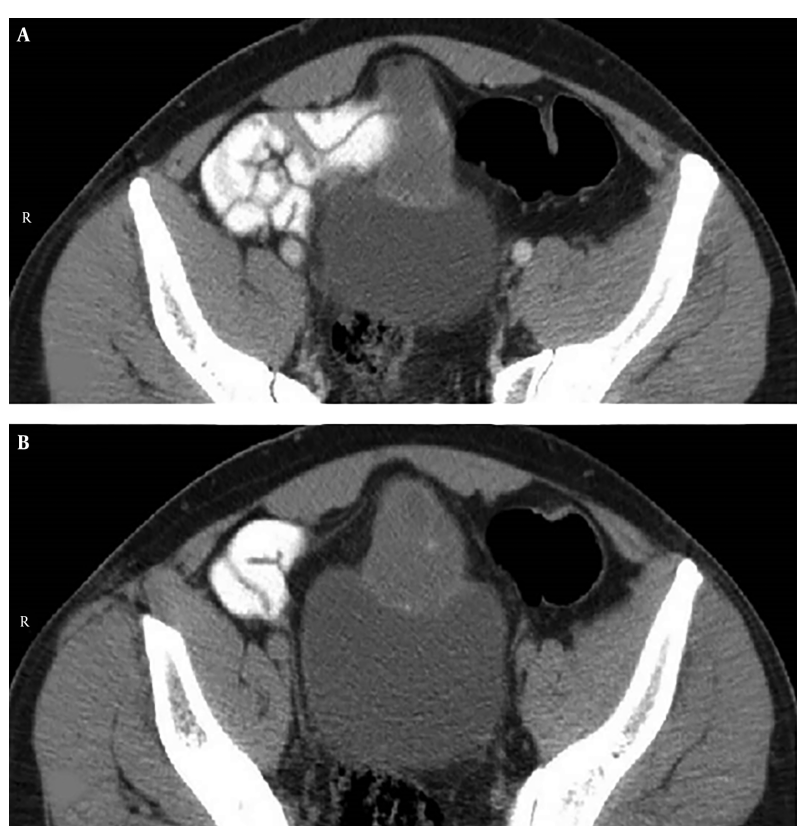

Figure 1. Axial image, contrast-enhanced CT scan of pelvis in the portal venous phase demonstrates heterogeneously enhancing mass lesion adjacent to bladder dome extending to umbilicus.

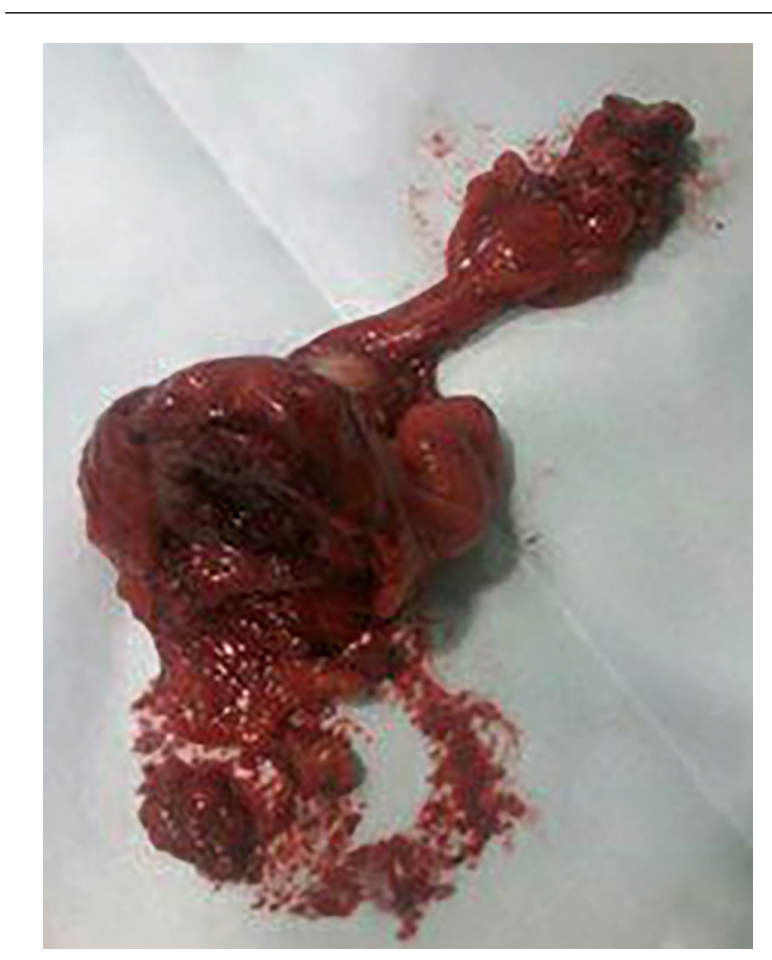

Figure 2. Tumor removed en bloc together with the umbilicus, lateral umbilical ligaments, adjacent peritoneum and bladder dome.

\begin{tabular}{ll}
\hline Table 1. The First and the Most Popular Clinical Staging System \\
\hline Stage & Definition \\
\hline Stage I & Urachal cancer confined to urachal mucosa \\
\hline Stage II & Urachal cancer with invasion confined to urachus itself \\
\hline Stage IIIA & Local urachal cancer extension to bladder \\
\hline Stage IIIB & Local urachal cancer extension to abdominal wall \\
\hline Stage IIIC & Local urachal cancer extension to peritoneum \\
\hline Stage IIID & Local urachal cancer extension to viscera other than bladder \\
Stage IVA & Metastatic urachal cancer to lymph nodes \\
\hline Stage IVB & Metastatic urachal cancer to distant sites \\
\hline
\end{tabular}

incision (Figure 2).

Histopathology of the material demonstrated urachal signet ring cell adenocarcinoma, with intact surgical borders. There was perivesical adipose tissue infiltration and regional lymph node metastasis (Sheldon stage IV A) (Figure 3). He was followed up with 3-month-interval cystoscopies and imaging up to 2 years with no localor distant relapse.

\section{Discussion}

The urachus is the embryological remnant of allantois and cloaca. It has patency with the urinary bladder, which would be obliterated with advancing age. However, its patency may persist in some patients (6).

Hue and Jacquin had presented urachal tumors for the first time in the 19th century. They consist of less than $1 \%$ of all bladder cancers and about 30\% of primary adenocarcinomas (7).

The MD Anderson Cancer Center (MDACC) introduced five criteria for urachal cancers, which include a midline mass, a distinct margin between the tumor and bladder urothelium, a bowel histology, no bladder dysplasia, cystitis cystica, or cystitis glandularis, as well as no adenocarcinoma in other sites $(8,9)$. In addition, Wheeler and Hill, in the 20th century, introduced five criteria: Mass in the anterior wall, inner extension of tumor in the bladder wall, no cystitis cystica or cystitis glandularis, presence of embryonic remnants, and no extra vesical origin (10). Our case fulfilled most of these two groups of criterias.

Sheldon et al. (1) had suggested the first and the most popular clinical staging system in 1984 (Table 1).

They are controversies in treatment of the urachal carcinomas. Surgical management includes resection of three parts: Umbilicus, urachal ligament, and the bladder (partial or total cystectomy). Partial cystectomy is the gold standard when the tumor is resectable (8). 

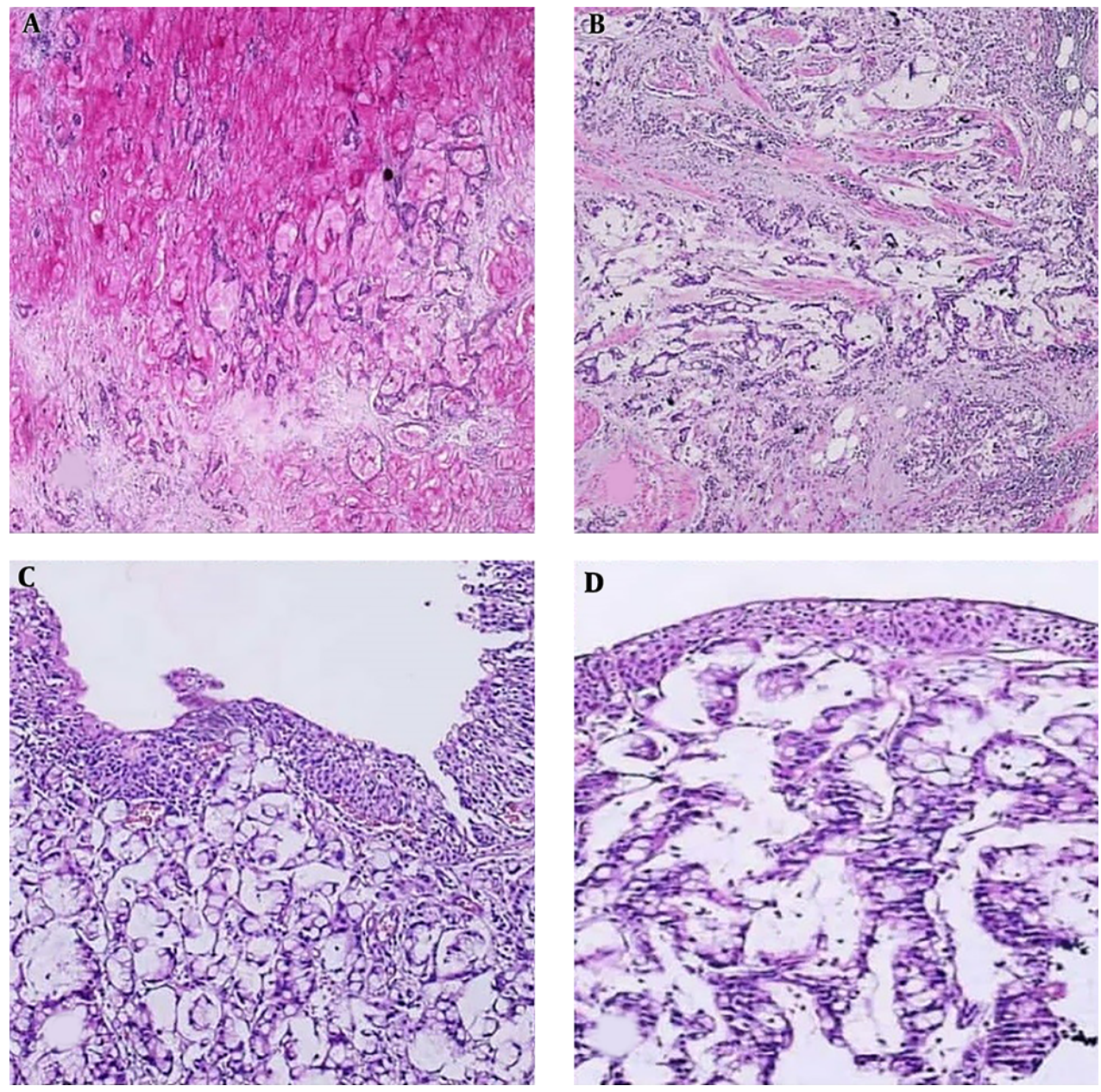

Figure 3. A, bladder mucosa infiltrated by a mucinous adenocarcinoma, $\mathrm{H} \& \mathrm{E}(\times 100)$. B, another view of the tumor showing obvious glandular differentiation with signet ring cells $(\times 100)$. C, invasion of the tumor through muscularis propria into bladder adventitia $(\times 40)$. D, PAS staining demonstrating intra and extra cellular mucin produced by the tumor $(\times 40)$.

In comparison with laparoscopic and robotic procedures, open surgical methods are the main treatment option due to the fact that there are no large patient series available to compare the results. Therefore, there has not been any definitive evidence of the usefulness of neo adjuvant or adjuvant chemotherapy in urachal adenocarcinoma.

For metastatic disease entsr inoperable pato, chemotherapy has shown poor usefulness in the management of urachal tumors, traditionally (11).

Prognostic factors for decreased survival include distant metastasis, local invasion to the abdominal wall, peritoneum or adjacent viscera, and lymph node involvement (12). In addition, one of the most significant prognostic factors is the status of surgical margins (12).
Ogaya Pinies et al. reported two cases of urachal adenocarcinomas, which were treated as extended partial cystectomy, the same as our reported case and stated that the main predictors of disease-free survival are the degree of tumor differentiation and the free margins of the surgical specimen (13).

Bao et al. reported a woman with urachal adenocarcinoma and involvement of ovaries, who underwent partial cystectomy and bilateral salpyno-ophoorectomy, without adjuvant therapy. In a duration of 5 years follow up, no recurrence or distant metastasis had been noticed (14).

Ashley et al. evaluated 130 patients with urachal mass between 1956 and 2004 at the Mayo Clinic. Among them, 70 patients had an urachal carcinoma, 60 patients underwent surgery, and $18 \%$ of the patients received adjuvant 
therapies due to nodal involvement or positive surgical margins. The adjuvant therapies consisted of multiagent chemotherapy in 3 patients, EBRT in 6 patients, and combination of chemoradiation in 3 patients. The authors stated that none of these adjuvant therapies was associated with a better outcome and the use of adjuvant therapy was not associated with a significant improvement in cancer-specific survival in their study (15).

In a series reported by Gopalan et al., three out of 24 patients received adjuvant radiotherapy, however, all three developed local recurrence in the follow up period (5).

The study of Chen et al. consisted of 14 patients $(64.3 \%$ of males (9/14) and 36.7\% females), which 92.9\% (13/14) of their resected urachal tumors proved to be adenocarcinoma. Seven patients in their study underwent extended partial cystectomy. Only one case had local recurrence two years after operation and the other six cases were free of recurrence 54 months later. One patient in their study was found to have rectus abdominis muscle involvement; therefore, part of the rectus abdominis was excised, too. Three patients in their study had advanced stages and were treated with chemotherapy alone. Two were followed up for seven and eight months after treatment and showed stable disease; however, one died six months after chemotherapy. Two patients had post-operative recurrence. One survived 21 months after combined therapy; while the other one did not receive any chemoradiotherapy and died three months after recurrence. They concluded that combined therapy has some impact on the patients' survival who has advanced disease and/or postoperative recurrence (16).

\subsection{Conclusion}

Urachal tumor is a rare and aggressive cancer. The optimal management of these tumors is uncertain due to its rarity. The main treatment method for urachal carcinoma is complete surgical resection. Chemotherapy has been considered for cases with distant metastatic disease or local invasion. We present a stage pT4a urachal adenocarcinoma, in a 35-year-old man, who underwent partial cystectomy and pelvic lymph node dissection with no systemic adjuvant therapy. In the period of the two year follow up, no local recurrence or distant metastasis was noticed.

\section{References}

1. Sheldon CA, Clayman RV, Gonzalez R, Williams RD, Fraley EE. Malig nant urachal lesions. J Urol.1984;131(1):1-8. [PubMed: 6361280].

2. Wright JL, Porter MP, Li CI, Lange PH, Lin DW. Differences in survival among patients with urachal and nonurachal adenocarcinomas of the bladder. Cancer.2006;107(4):721-8. doi:10.1002/cncr.22059. [PubMed: 16826584].

3. Marquez Moreno AJ, Gomez Rebollo C, Antuna Calle F, Perez Villa L, Sanchez Carrillo JJ, Julve Villalta E, et al. [Urachal adenocarcinoma with late brain metastases]. Arch Esp Urol. 2010;63(7):550-4. English, Spanish. [PubMed: 20945591].

4. Schnyder P, Candardjis G. Vesicourachal diverticulum: CT diagnosis in two adults. AJR Am J Roentgenol. 1981;137(5):1063-5. doi: 10.2214/ajr.137.5.1063. [PubMed: 6794339].

5. Gopalan A, Sharp DS, Fine SW, Tickoo SK, Herr HW, Reuter VE, et al. Urachal carcinoma: A clinicopathologic analysis of 24 cases with outcome correlation. Am J Surg Pathol. 2009;33(5):659-68. doi: 10.1097/PAS.ob013e31819aa4ae. [PubMed: 19252435]. [PubMed Central: PMC4225778].

6. Mardi K, Gupta N. Urachal papillary cystadenocarcinoma: A rare case report. J Cancer Res Ther. 2011;7(2):223-5. doi: 10.4103/0973-1482.82934. [PubMed: 21768722].

7. Munichor M, Szvalb S, Cohen H, Bitterman W. Mixed adenocarcinoma and neuroendocrine carcinoma arising in the urachus. A case report and review of the literature. Eur Urol.1995;28(4):345-7. [PubMed: 8575505]

8. Siefker-Radtke A. Urachal carcinoma: Surgical and chemotherapeutic options. Expert Rev Anticancer Ther. 2006;6(12):1715-21. doi 10.1586/14737140.6.12.1715. [PubMed: 17181485].

9. Siefker-Radtke AO, Gee J, Shen Y, Wen S, Daliani D, Millikan RE, et al. Multimodality management of urachal carcinoma: The M. D. Anderson Cancer Center experience. J Urol. 2003;169(4):1295-8. doi: 10.1097/01.ju.0000054646.49381.01. [PubMed: 12629346].

10. Wheeler JD, Hill WT. Adenocarcinoma involving the urinary bladder. Cancer. 1954;7(1):119-35. doi: 10.1002/1097-0142(195401)7:1<119::aidcncr2820070113>3.0.co;2-8.

11. Logothetis CJ, Samuels ML, Ogden S. Chemotherapy for adenocarcinomas of bladder and urachal origin: 5-fluorouracil, doxorubicin, and mitomycin-C. Urology. 1985;26(3):252-5. [PubMed: 3929441].

12. Herr HW, Bochner BH, Sharp D, Dalbagni G, Reuter VE. Urachal carcinoma: Contemporary surgical outcomes. J Urol. 2007;178(1):74-8. discussion 78. doi: 10.1016/j.juro.2007.03.022. [PubMed:17499279].

13. Ogaya Pinies G, Herranz Amo F, Escribano Patino G, Lledo Garcia E, Molina Escudero R, Husillos Alonso A, et al. Urachal adenocarcinoma. Case report and bibliographic review. Arch Esp Urol. 2012;65(4):498501. [PubMed: 22619143].

14. Bao B, Hatem M, Wong JK. Urachal adenocarcinoma: A rare case report. Radiol Case Rep. 2017;12(1):65-9. doi: 10.1016/j.radcr.2016.10.019. [PubMed: 28228881]. [PubMed Central: PMC5310256].

15. Ashley RA, Inman BA, Sebo TJ, Leibovich BC, Blute ML, Kwon ED, et al Urachal carcinoma: Clinicopathologic features and long-term outcomes of an aggressive malignancy. Cancer. 2006;107(4):712-20. doi: 10.1002/cncr.22060. [PubMed:16826585].

16. Chen ZF, Wang F, Qin ZK, Dai YP, Zhou FJ, Han H, et al. [Clinical analysis of 14 cases of urachal carcinoma].Ai Zheng. 2008;27(9):966-9. Chinese. [PubMed: 18799037]. 